\title{
CAN A BRIEF ANESTHESIOLOGIST INTERVENTION MODIFY SMOKING BEHAVIOUR?
}

Christopher Pysyk, BSc, Student, Faculty of Medicine and Dentistry, University of Alberta, Edmonton, Alberta, T6G 2R7

Barry Finegan PhD, MB, FRCPC, Department of Anesthesiology, University of Alberta Hospital, Edmonton, Alberta, T6G 2B7

T. Cameron Wild PhD, Department Of Public Health Sciences, University Of Alberta, Edmonton, Alberta, T6G 2G3

\section{INTRODUCTION}

Smoking is associated with an increase in wound infection and pulmonary complications following surgery ${ }^{1}$. The preoperative anesthetic assessment clinic visit provides a unique opportunity to modify smoking behaviour through counselling, in a motivated population of patients who postoperatively undergo obligatory cessation as a consequence of inpatient admission. In this pilot study we sought to determine the demographics and smoking characteristics of surgical patients who smoke and assess the effectiveness of counselling by the anaesthesiologist in reducing self-reported long-term tobacco consumption.

\section{METHODS}

Following ethical approval, patients identified as smokers were recruited to participate in a singlegroup prospective study. After completing a baseline questionnaire assessing socio-demographics, nicotine dependence and quitting motivation, participants were briefly advised by the anaesthesiologist about smoking cessation, provided with literature and a business card for a local smoking cessation helpline. A six-month telephone follow-up was used to obtain self-reported current smoking behaviour and quit attempts since surgery.

\section{RESULTS}

To date, data (mean $\pm \mathrm{SD}$ ) from 79 participants (57\% male) have been analysed: age $50 \pm 12$ years; number of years since started smoking regularly $31 \pm 12$ and Fagerstrom nicotine dependence score $4.6 \pm 2.2$. Logistic regression analysis indicated that the only significant predictor of quitting was participant age $(O R=1.13, p<.05)$. Correlational analyses indicated that participant age was positively correlated with beliefs that hospitalization was related to smoking status $(r=.31, p<.01)$, and perceived importance of their physician in their decision to quit or reduce cigarette use $(r=.24, p<.05)$. Thirteen subjects reported not smoking at follow-up (16.5\% quit rate).

\section{DISCUSSION}

The demographic and dependence data in this sample are comparable to the community as a whole. Our data suggest that, for some patients, the preoperative visit is a particularly appropriate time for brief counselling about long term smoking cessation. The quit rate we observed, though modest, was comparable to other hospital-based cessation programmes. ${ }^{2}$. These data suggest that beneficial longterm risk modification is possible in a subgroup of surgical patients through the development of organized smoking cessation programs in preadmission clinics.

\section{REFERENCES}

$1 \quad$ Anesthesiology 2000; 97:842

$2 \quad$ Prev Med. 2004; 39:1087 